physical discomfort, being a bully victim, having a special healthcare need, and being overweight.

Conclusions: Inthis longitudinal study, characteristic patterns of child health predicted future academic achievement. These findings suggest that health promotion efforts provided to children in elementary school may have beneficial effects on academic performance in adolescence.

160

\section{CLUSTER RANDOMISED TRIAL OF AN INFORMATION LEAFLET DESIGNED TO PROMOTE SELF-MANAGEMENT OF HIVIAIDS IN ZAMBIAN ADOLESCENTS}

\author{
S. Degun ${ }^{1}$, E. Atkins ${ }^{1}$, A. Menon ${ }^{2}$, R. Nair ${ }^{3}$, \\ P. Garrud ${ }^{4}$, A. McPherson ${ }^{5}$, C. Glazebrook ${ }^{1}$
}

${ }^{1}$ Psychiatry, University of Nottingham, Nottingham, UK, ${ }^{2}$ Psychology, University of Zambia,

Lusaka, Zambia, ${ }^{3}$ Institute of Work Health and Organisations, ${ }^{4}$ Graduate Entry Medicine, University of Nottingham, Nottingham, UK, ${ }^{5}$ Bloorview Kids Rehab, Toronto, ON, Canada

Objective: The study aimed to examine whether an information leaflet designed to promote selfmanagement of health in adolescents with HIV/ AIDS was effective in increasing knowledge about HIV, reducing perceived stigma associated with the disease and increasing sense of control in Zambian school children.

Method: In this cluster randomised controlled trial, 8 schools in the Lusaka region of Zambia were randomised to receive either an English language HIVIAIDS information leaflet $(n=156)$ or a control leaflet $(n=186)$. Inclusion criteria were age 11-16 years and attending Grades 8 or 9 in the study schools. Students were given the leaflet and encouraged to discuss its content with their peers. The leaflets were removed and students completed measures of knowledge, stigma and health locus of control. Researchers analysing the data were blind to leaflet allocation.

Results: Participants receiving the HIVIAIDS leaflet $(n=156)$ had higher levels of knowledge about HIV/ AIDS $(p<0.001$, Cohen's d'=0.82); a greater sense of control over health $(p<0.05$, d'=0.24), and lower levels of stigma associated with HIVIAIDS $(p<0.001$, $\left.d^{\prime}=0.55\right)$ compared to those receiving the control leaflet $(n=186)$. Better HIVIAIDS knowledge was associated with lower stigma $(r=-0.373, p<0.001)$ and, controlling for other factors, $21 \%$ of variance in stigma scores was accounted for by HIVIAIDS knowledge $(p<0.001)$.

Conclusion: A brief educational intervention was effective in improving knowledge about HIV/ AIDS and reducing stigma in a normal population of Zambian adolescents. The results support the use of the leaflet in peer education and in clinical samples to promote self-care.

\section{1}

PRENATAL NICOTINE EXPOSURE INCREASES THE RISK OF NEONATAL APNEA -A NATIONAL BIRTH-COHORT STUDY

\author{
A. Gunnerbeck ${ }^{1}$, A.-K. Wikström², \\ A.-K.E. Bonamy ${ }^{1}$, R. Wickström ${ }^{1}$, S. Cnattingius ${ }^{3}$ \\ ${ }^{1}$ Neonatal Research Unit, Dep.of Women's and \\ Children's Health, Karolinska Institutet, Stockholm, \\ ${ }^{2}$ Dep. of Women's and Children's Health, \\ Obstretics and Gynecology, Uppsala University, \\ Uppsala, ${ }^{3}$ Dep.of Medicine, Unit of Clinical \\ Epidemiology, Karolinska Institutet, \\ Stockholm, Sweden
}

Background: Smoking during pregnancy is associated with disturbed cardio-respiratory control of the infant and is a major risk factor for sudden infant death syndrome (SIDS). Although it is not known whether the harmful effects of smoking are caused by nicotine or other toxic substances in tobacco smoke, nicotine replacement therapy (NRT) during pregnancy is often regarded as being safer than smoking.

Aim of study: To investigate if the disturbances in cardio-respiratory control seen in infants exposed to smoke are also noticed in infants exposed to nicotine (in the form of snuff) prenatally.

Method: We conducted a population-based cohort study in the Swedish Medical Birth Register, 19992006, to study the association between maternal tobacco use and neonatal apnea.

Of 569,859 newborns, 7,599 were born to snuffusing mothers, 41,391 and 16,928 born to light smokers (1-9 cig./day) and heavy smokers ( $\geq 10$ cig./day), respectively. Apnea was defined by using ICD-10 codes P28.2-P28.4. Logistic regression was used for statistical analysis.

Results: Compared with infants to non-users, prenatal exposure to snuff doubled the risk of apnea, and this risk was not confounded by maternal or 